\title{
Finding the Incidence of Ventilator Associated Pneumonia by Recent NHSN Guidelines and Its Bacteriological Profile: A Study Conducted in a Tertiary Care Hospital in Southern India
}

\author{
Sadiya Fatima $^{1 *}$, S. Rajeshwar Rao ${ }^{2}$, V.V. Shailaja ${ }^{3}$ and K. Nagamani ${ }^{4}$ \\ Department of Microbiology, Gandhi Medical College and Hospital, Secunderabad, \\ Telangana, India \\ *Corresponding author
}

Ke y w or d s
Intensive care unit,
Mechanical
ventilation (MV),
Ventilator
associated event,
Ventilator
associated
pneumonia
Article Info
Accepted:
15 September 2019
Available Online:
10 October 2019

Ventilator associated pneumonia is the second most common nosocomial infection in the intensive care unit (ICU) and the most common in mechanically ventilated patients. The present study was undertaken to elucidate the bacteriological profile causing VAP in our institution and finding its incidence by recent NHSN guidelines. Study was conducted for 1 year study period (June 2017- May 2018). All the patients were monitored from the time of inclusion in the study for the entire duration of the hospital stay. Relevant details of the patients were included in the study in a structured proforma and surveyed for possible VAP as per the recent NHSN guidelines. Gram stain and semi-quantitative cultures of Purulent Endotracheal aspirates of patients were processed as per standard protocols. The clinical isolates obtained were identified by both conventional and automated methods. Among 104 patients 31 developed PVAP (possible VAP) during their ICU stay; of these two patients had 2 episodes of VAP each, incidence of VAP was $32 \%$. The overall incidence rate was $38.42 / 1000 \mathrm{VD}$. Most common isolate was Acinetobacter baumani (38\%) followed by Pseudomonas aeruginosa (22\%), Klebsiella pneumoniae (16\%) and Escherichia coli $(13.51 \%)$. The overall mortality was $48.38 \%$. There is a need for compilation of local epidemiological data at all centers, as such information can help in guiding the initial empirical therapy which would reduce the ICU stay thereby the rate of VAP.

\section{Introduction}

Ventilator associated pneumonia refers to bacterial pneumonia developed in patients who have been mechanically ventilated for a duration of more than $48 \mathrm{hrs} .{ }^{1}$ It is the second most common nosocomial infection in the intensive care unit (ICU) and the most common in mechanically ventilated patients. The incidence of VAP ranges from 13 to 51 per 1000 ventilator days. ${ }^{2}$

The incidence of VAP varies among different studies, depending on the definition, the type of hospital or ICU, the population studied, and the level of antibiotic exposure. ${ }^{3}$ The causative 
organisms vary according to the patients demographics in the ICU, the duration of hospital/ICU stay, and the antibiotic policy of the institution.

The study was conducted to find the incidence of PVAP by using the recent definition guidelines and to elucidate bacteriological profile of VAP among mechanically ventilated patients admitted in RICU department of Gandhi Hospital. Acinetobacter spp., Pseudomonas spp, Escherichia coli, Klebsiella pneumoniae, and Staphylococcus aureus were identified as the common VAP pathogens

Although mechanical ventilation (MV) is a life-saving intervention, it has its own potential complications. VAP occurrence is increased with prolonged length of ICU stay ${ }^{04,05}$ A method to reduce the risk of VAP is to extubate patients as soon as possible as various randomized, and observational studies have shown that the risk of developing VAP increases with the duration of an endotracheal tube remaining in place. $^{06}$ The use of appropriate weaning protocols and the regular assessment of sedation requirements are effective in reducing the duration of MV and hence the incidence of VAP. ${ }^{07}$

\section{Materials and Methods}

\section{Setting and subjects}

The prospective study was conducted over a period of 1 year from June-2017 to May 2018 of all mechanically ventilated patients admitted in RICU of Gandhi medical college and hospital a tertiary care hospital in Telangana, India.

An ethical clearance to conduct this study was obtained from institutional ethical committee prior to commencement of the study.

The subjects consisted of all adult patients (>18yrs) presented with acute respiratory failure due to a variety of causes and required mechanical ventilation for $>48$ hours.

Patients not admitted in RICU (Respiratory Intensive care units) i.e. admitted in general wards, other ICU's or treated in other departments, Patients with pneumonia prior to MV or within 48 hours of MV and Patients on high frequency ventilation or extracorporeal life support or brain dead, Lung expansion devices such as intermittent positive-pressure breathing (IPPB), Nasal positive endexpiratory pressure (nasal PEEP), Continuous nasal positive airway pressure (CPAP, hypo CPAP) ${ }^{08}$ were excluded.

\section{Study design and data collection}

All the relevant details of the patients included in the study, i.e. name, age, sex, occupation, diagnosis, duration of illness, reason for mechanical intubation, whether any surgical intervention done, history of antibiotic usage, site of infection, past history, family history, were taken in a structured proforma.

\section{Procedure for data collection}

All patients included in the study were monitored daily for the development of VAP using recent CDC NHSN clinical and microbiological criteria until either discharge or death.

The clinical parameters were recorded from their medical records and bedside charts. Details of antibiotic therapy, surgery, use of steroids, duration of hospitalization, presence of neurological disorders, and impairment of consciousness were also noted

\section{Criteria for diagnosis of VAP}

Oxygen demand on ventilator was measured by fraction of inspired oxygen (FiO2) or positive end-expiratory pressure (PEEP). 


\section{Criteria for defining VAC}

Ventilator associated condition is defined as worsening of oxygenation sustained for at least $2 \mathrm{CL}$ immediately after the baseline period of stability or improvement of 2 days.

Worsening of oxygenation defined as

FiO2: $\uparrow$ in daily minimum FiO2 of $\geq 0.20$ (20\%) after 2 calendar days ofstability (OR)

PEEP: $\uparrow$ in the daily minimum PEEP of $\geq 3 \mathrm{~cm} \mathrm{H} 2 \mathrm{O}$ after 2 calendar days of stability (PEEP values of $0 \mathrm{~cm}-5 \mathrm{~cm} \mathrm{H} 2 \mathrm{O}$ are considered equivalent)

\section{Criteria for defining IVAC}

Both of the criteria must occur in the VAE window period

$\begin{aligned} & \square \text { Presence of temperature }>38^{\circ} \mathrm{C} \text { or }<36^{\circ} \mathrm{C} \text { or } \mathrm{WBC} \geq 12,000 \text { cells } / \mathrm{mm} 3 \text { or } \leq 4000 \\ & \text { cells } / \mathrm{mm} 3 \\ & \text { AND } \\ & \square \text { A new antimicrobial agent }(\mathrm{s})^{*} \text { is started and continued for } \geq 4 \text { calendar days in a } \\ & \text { mechanically ventilated patient on or after calendar day } 3\end{aligned}$

Criteria for defining Possible VAP (PVAP) as microbiological evidence of infection in patient with IVAC.

$\square$ Culture without sufficient growth having Purulent respiratory secretions (>25 neutrophils and $<10$ squamous epithelial cells per low power field)

\section{Microbiological techniques}

\section{Specimen collection}

Endotracheal aspirate (ETA) was chosen as sample because it is non-invasive and was proved to give similar results when compared with invasive procedures like PSB (Protected specimen brush), BAL (Broncho alveolar lavage).The ETA was collected under aseptic precaution in the patient qualifying IVAC criteria using a 22- inch Ramson's 12 F suction catheter with a mucus extractor (Lukens trap shown in the figure 1), which was gently introduced through the endotracheal tube for a distance of approximately $25-26 \mathrm{~cm}$.

\section{Specimen processing}

Specimen was immediately processed after collection. Gram stain of the sample was done ${ }^{09}$.

To consider it as a purulent sample, Gram stain should show : >25 PMN neutrophils/LPF and $<10$ squamous epithelial cells. One of those purulent gram stain is shown in figure 2 . Semi-Quantitative cultures were done by serial dilution in sterile normal saline as $1 / 10$, $1 / 100,1 / 1000$, and $0.01 \mathrm{ml}$ of $1 / 1,000$ dilution was inoculated on 5\% sheep blood agar, Chocolate agar, MacConkey agar and Sabourad's Dextrose agar. Inoculated plates were incubated at $37{ }^{0} \mathrm{C}$ for 18-24 hrs .All 
plates were checked for growth overnight and then after 24-48 hr of incubation. SDA slants were checked up for 4 weeks. Colony count was done and expressed as number of colony forming units per $\mathrm{ml}(\mathrm{CFU} / \mathrm{ml})$, The microorganisms isolated at a concentration of more than $10^{5} \mathrm{CFU} / \mathrm{ml}$ were considered as significant and also if the colony count is less then purulent gram stain was taken into consideration and colonies were identified based on standard bacteriological procedures including colony morphology and biochemical reactions ${ }^{10}$. Subsequently Further confirmation of identification was done by automated Vitek2 system.

\section{Results and Discussion}

Over the 1 year study period (June 2017 to May 2018) 204 patients were admitted in the respiratory intensive care unit were prospectively evaluated. Of these 28 patients $(13.72 \%)$ were not intubated, as there were no indications for mechanical ventilation.

Among those requiring MV, 72 (35.29\%) patients were mechanically ventilated for less than 48 hours therefore excluded from the study.

\section{Incidence}

$104(50.98 \%)$ patients received mechanical ventilation for more than 48 hours and were monitored daily. Of these 104 patients, 31 (15.19\%) patients developed VAP during their ICU stay. 2 patients had 2 episodes of VAP each. Incidence of VAP was $31.73 \%$ as shown in Table 1.

Formula to calculate VAP rate:

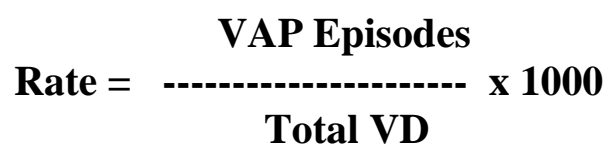

The overall incidence rate was 38.42 per 1000 ventilator days.

VAE was more in the patients staying for more than 10 days and it was less when the duration of mechanical ventilation was less. Number of patients was more in $<5$ days MV but the development of VAP was less though VAC was there. Patients on MV for >15days were less but most of them developed VAP signifying the role of duration of MVfor VAP.

The incidence of VAP was more common in males $(71 \%)$ than females $(29 \%)$ as shown in figure 3. Male sex was found to be one of the non-modifiable patient related risk factor for the development of VAP.

\section{Organism wise distribution of VAP}

Acinetobacter spp was the most common organism (37.83\%) among which Acinetobacte rbaumanii was more common than A. lowfii. Pseudomonas spp (21.62\%) were the second most common organism followed by Klebsiella spp (16.21\%), Escherichia coli (13.51\%) while Elizabethkingia meningoseptica and Enterobacter cloacae were the least common one among gram negative organisms being only one isolate $(2.70 \%)$ each. The 2 isolates of Staphylococcus aureus accounting for $(5.40 \%)$ were the only gram positive organism identified. No fungal isolate found in any of the sample tested (Fig. 4 and Table 2).

\section{Outcome}

In this study the crude mortality rate of patients with VAP was $48.38 \%$.

Novelty of our work comes from being the first to study VAP according to newer NHSN guidelines in Telangana by taking into consideration clinical, radiological and microbiological results together. VAP accounts for one-fourth of the infections 
occurring in critically ill patients and is the reason for half of antibiotic prescriptions in mechanically ventilated patients. Several countries have reported mortality rates ranging from $24 \%$ to $76 \%$ (Table 3 ).

Fig.1 Lukens trap

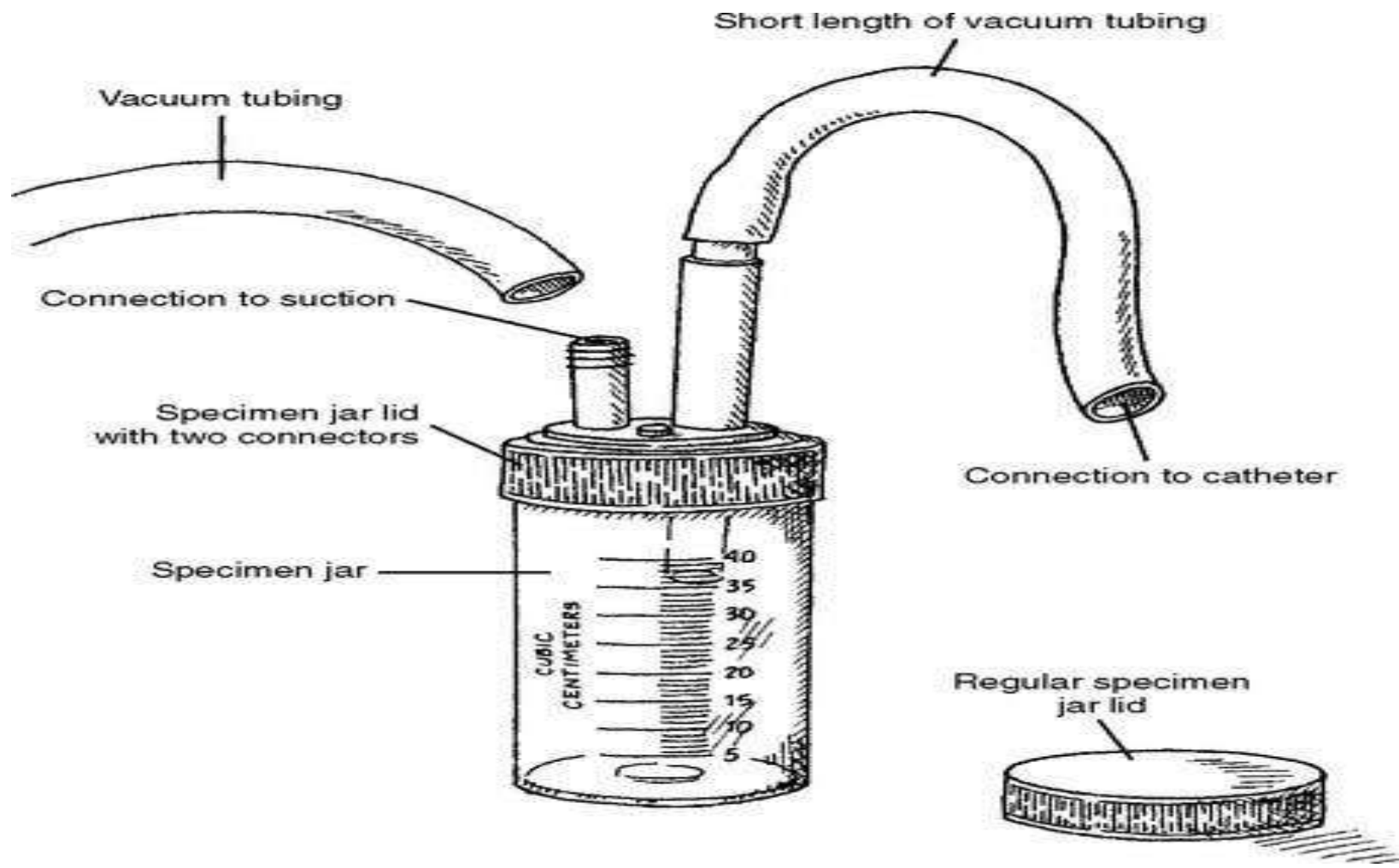

Fig.2 Direct Gram's stain smear showing plenty of polymorphonuclear leucocytes

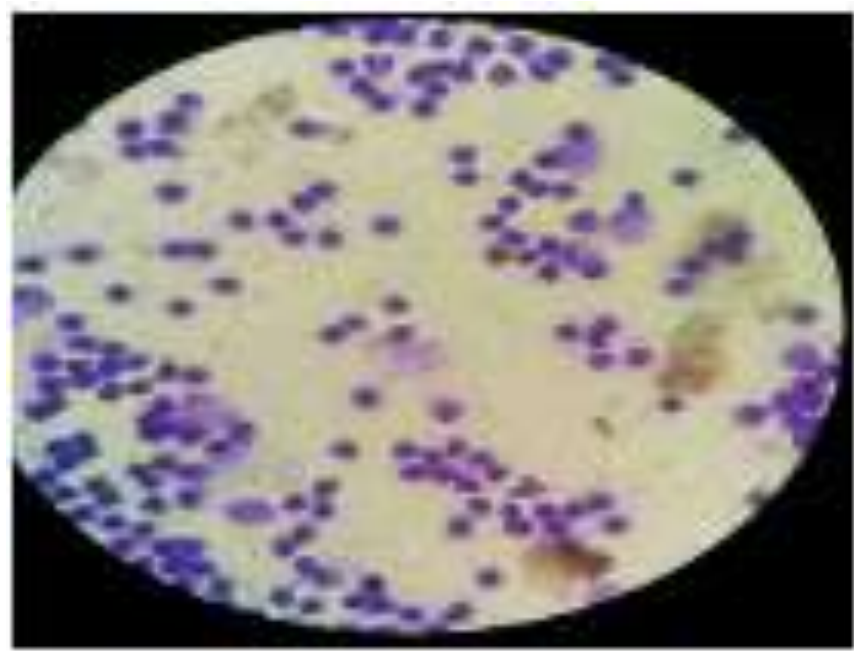


Table.1 Incidence of VAP

\begin{tabular}{|c|c|c|}
\hline Episodes of VAP & Patients & Total Patients \\
\hline 33 & 31 & 104 \\
\hline
\end{tabular}

Table.2 Overall VAP Rate

\begin{tabular}{|c|c|c|}
\hline VAP & Total Ventilator Days & Rate (Per 1000 VDs) \\
\hline 33 & $\mathbf{8 5 9}$ & $\mathbf{3 8 . 4 1 6}$ \\
\hline
\end{tabular}

Table.3 Correlation between ventilator days and development of ventilator-associated events

\begin{tabular}{|l|c|c|c|c|}
\hline \multirow{2}{*}{ VAE } & \multicolumn{4}{|c|}{ Ventilator days (VDs) } \\
\cline { 2 - 5 } & $\leq \mathbf{5}$ days & $\mathbf{6 - 1 0}$ days & $\mathbf{1 1 - 1 4}$ days & $>15$ days \\
\hline Number of patients on MV & $\mathbf{4 3}$ & $\mathbf{3 4}$ & $\mathbf{1 1}$ & $\mathbf{1 6}$ \\
\hline Episodes of VAC only & $\mathbf{1 3}$ & $\mathbf{1 9}$ & $\mathbf{1 0}$ & $\mathbf{1 2}$ \\
\hline Episodes of IVAC & $\mathbf{0 7}$ & $\mathbf{0 8}$ & $\mathbf{1 0}$ & $\mathbf{1 0}$ \\
\hline Episodes of PVAP & $\mathbf{0 4}$ & $\mathbf{0 8}$ & $\mathbf{1 0}$ & $\mathbf{1 1}$ \\
\hline $\begin{array}{l}\text { Episodes of PVAP per number } \\
\text { of patients }\end{array}$ & $\mathbf{9 . 3 0 \%}$ & $\mathbf{2 3 . 5 3 \%}$ & $\mathbf{9 0 . 9 \%}$ & $\mathbf{6 8 . 7 5 \%}$ \\
\hline
\end{tabular}

Fig.3 Male and Female distribution in VAP cases

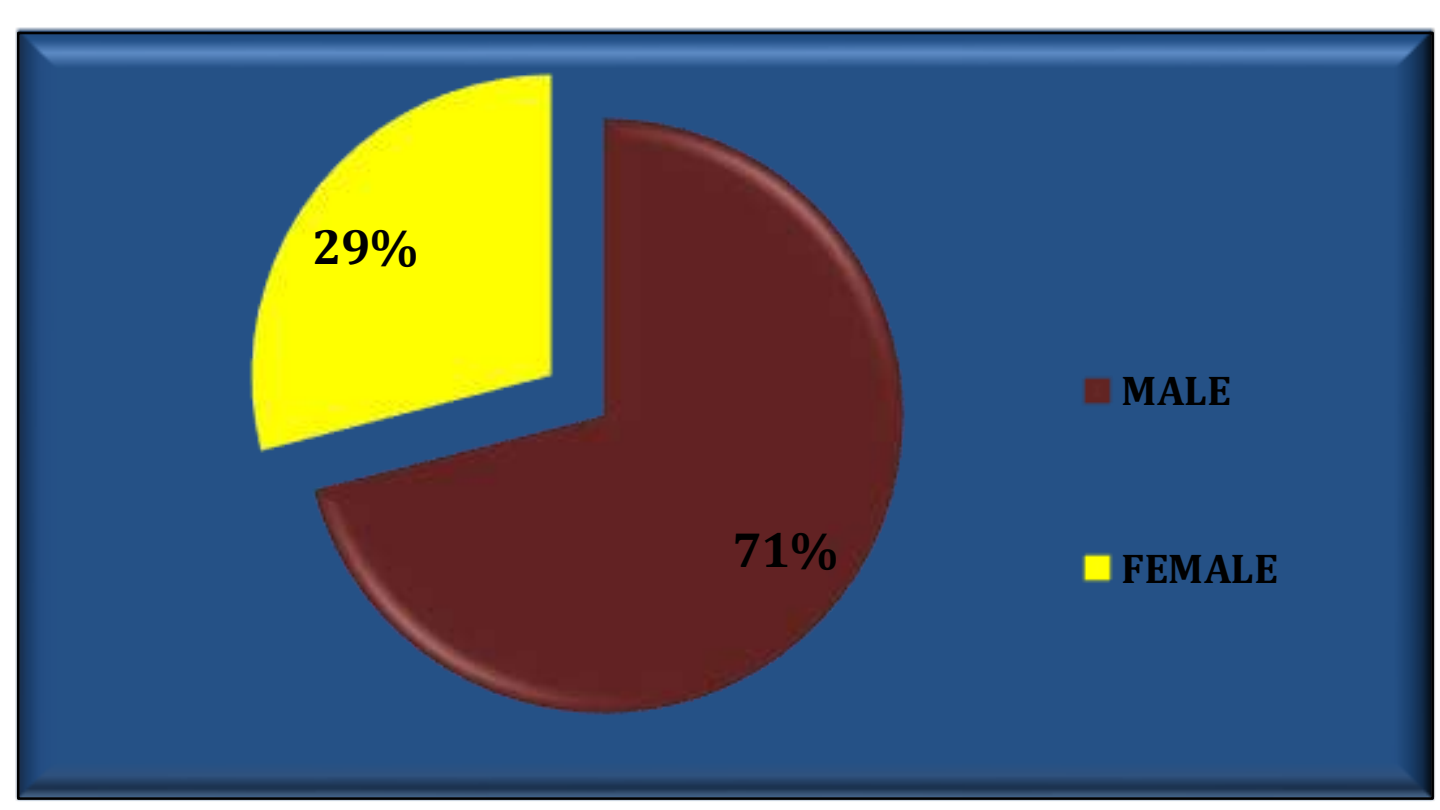


Fig.4 Organism wise distribution of VAP

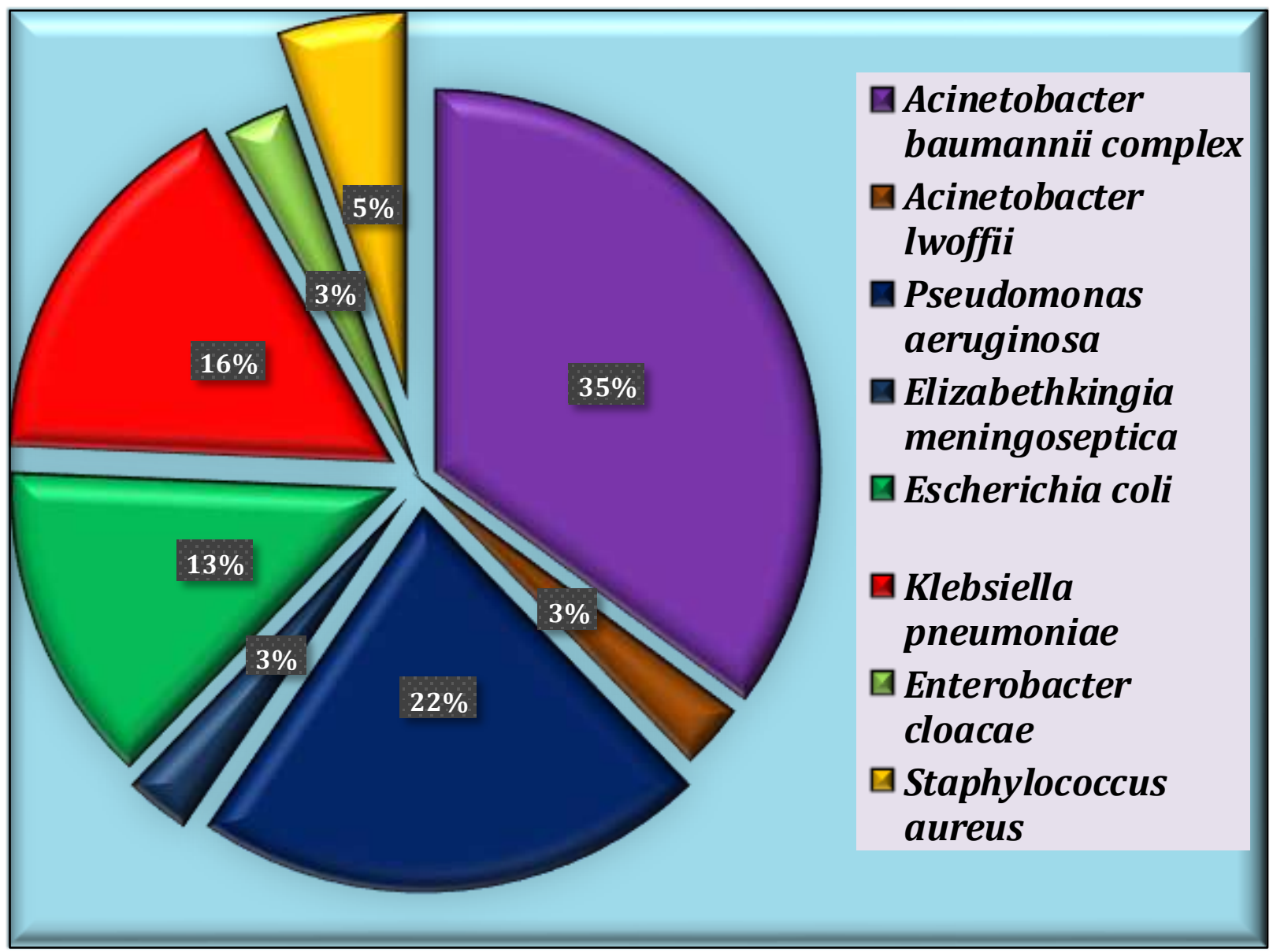

In Present study Incidence rate of VAP was $31.73 \%$ correlating with studies from Odisha by Mohanty, et al., $(2016)^{11}$ who reported as $30 \%$, from UP by Alok Gupta et al., $(2011)^{12}$ who reported as $28.04 \%$, from Saudi Arabia by Abdelrazik Othman et al., (2017) ${ }^{13}$ who reported as $35.4 \%$. While a study from MP by Ranjan et al., (2014) ${ }^{14}$ reported $57.14 \%$ and from Maharashtra by Deshmukh B et al., $(2017)^{15}$ reported $78 \%$. Divergence of incidence can be attributed to several factors such as differences in the study population, differences in the definition of VAP, e.g. depending on the diagnostic criteria used, clinically versus microbiologically oriented and possibly, to the use of preventive strategies and critical care practices in the ICUs.
Sex distribution in VAP cases in our study was found to $70.96 \%$ among male and female constituted $29.03 \%$. Vinitgarg et al., ${ }^{16}$ in 2017 reported male predominance around $68.3 \%$ and SarojGolia et al., ${ }^{17}$ in 2013 also found incidence of VAP is more in men $(65.4 \%)$ than females $(34.61 \%)$. Usman et al., $(2014)^{18}$ also reported male dominance $(65 \%)$ in his study.

Acinetobactersps followed by Pseudomonas aeruginosa, Escherichia coli and Klebsiella pneumonia were common organisms isolated in this study. The organisms implicated in VAP were similar in other studies such as Dube et al., (2018) $)^{19}$, Maqbool et al., $(2017)^{20}$, Mathai et al., (2016) ${ }^{21}$ and Ranjan et al., (2014) $)^{22}$ with Acinetobacterspsas the most 
common organism isolated. In contrast Deshmukh et al., Masih et al., $(2016)^{23}$ and Husain Shabbir Ali et al., $(2016)^{24}$ reported Pseudomonas aeruginosa as the most common organism.

In our study mortality was $48.38 \%$ and it is consistent with the recent reports from Dube et al., Maqbool et al., Ranjan et al., Goel et al., $(2012)^{25}$ and Gupta et al., $(2011)^{26}$. Higher mortality was reported by Gupta et al., as $78.94 \%$. Lower mortality was reported by Kant et al., (2015) ${ }^{27} 15.3 \%$ and Patil and Patil et al., $(2017)^{28} 29.72 \%$. This vast difference in the mortality rate may be attributed to the management of the cases by treatment and preventive measures taken and also the associated comorbidities associated with the patients.

The notable strengths of our study are that it was prospectively conducted, with the diagnosis of VAP based on new NHSN guidelines including clinical, radiological and microbiological results. To date, most Indian studies on VAP infections are from a laboratory-based perspective or considering CPIS scoring system.

This study highlights the need for urgent infection control, planning, as well as multidisciplinary team participation to combat VAP. This includes implementing measures such as education, increased awareness of hand hygiene measures, reduction of the duration of mechanical ventilation and use of other VAP bundles, all of which have been proven to reduce the risk of VAP infections.

Regarding limitations of this study, Findings emerging out of this study may not be generalized as a single centre study limits the generalizability of the findings to other regions of the country. More studies with bigger sample size are warranted.
In conclusion, the findings showed VAP as a problem in the ICU setting, with high percentage of gram negative pathogens and high mortality. Further, to have a comprehensive pan-India picture, multicentric studies with high number of patient population need to be initiated. Majority of these are caused by highly resistant strains and also the frequency of specific pathogens causing VAP may vary by hospital, patient population, and exposure to antibiotics, type of ICU patients and changes over time, emphasizing the need for timely local surveillance data. Adherence to the best practices standards of hospital infection control requires an interdisciplinary team of clinical microbiologists, physicians and hospital infection control nurses, to collectively manage these patients.

\section{References}

1. Davis K A. Ventilator-associated pneumonia: a review. J Intensive Care Med. 2006; 21:211-26.

2. Torres A, Ferrer M, Badia JR. Treatment guidelines and outcomes of hospital-acquired and ventilator-associated pneumonia. Clin Infect Dis 2010; 51Suppl 1:S48-53.

3. Masih SM, Goel S, Singh A, Tank R, Khichi SK, Singh S. Incidence and risk factors associated with development of ventilator- associated pneumonia from a tertiary care center of northern India. Int J Res Med Sci. 2016; 4: 1692-7.

4. Bercault N, Boulain T. Mortality rate attributable to ventilator-associated nosocomial pneumonia in an adult intensive care unit: A prospective case control study. Crit Care Med 2001; 29: 2303-9.

5. Heyland DK, Cook DJ, Griffith L, Keenan SP, Brun-Buisson C. The attributable morbidity and mortality of ventilator-associated pneumonia in the 
critically ill patient. The Canadian critical trials group. Am J RespirCrit Care Med 1999; 159: 1249-56.

6. Cook D, De Jonghe B, Brochard L, Brun-Buisson C. Influence of airway management on ventilator-associated pneumonia: Evidence from randomized trials. JAMA 1998; 279: 781-7

7. Quenot JP, Ladoire S, Devoucoux F, Doise JM, Cailliod R, Cunin N, et al. Effect of a nurse-implemented sedation protocol on the incidence of ventilatorassociated pneumonia. Crit Care Med 2007; 35: 2031-6.

8. National Healthcare Safety Network (NHSN) Patient Safety Component Manual chapter 10: ventilator associated event (VAE)

9. Colle JG, Fraser AG, Marmion BP, Simmons A. Mackie \& McCartney Practical Medical Microbiology: staining methods $14^{\text {th }}$ ed. New Delhi: Reed Elsevier India Private Limited; 2016. p.793-812.

10. Mackie TJ and McCartney JE (1996) Practical medical microbiology, 14th edition. New York: Churchill Livingstone 978p.

11. Debaprasad Mohanty, Sidharth Sraban Routray, Debasis Mishra, Abhilas Das. Ventilator associated pneu-monia in a ICU of a tertiary care hospital in India. International Journal of Contemporary Medical Research 2016;3(4):10461049.

12. Gupta A, Agrawal A, Mehrotra S, Singh A, Malik S, Khanna A. Incidence, risk stratification, antibiogram of pathogens isolated and clinical outcome of ventilator associated pneumonia. Indian $\mathbf{J}$ Crit Care Med 2011; 15: 96-101.

13. A. Abdelrazik Othman, M. Salah Abdelazim. Ventilator-associated pneumonia in adult intensive care unit prevalence and complications .The
Egyptian Journal of Critical Care Medicine 5 (2017) 61-63

14. Ranjan N, Chaudhary U, Chaudhry D, Ranjan KP. Ventilator-associated pneumonia in a tertiary care Intensive Care Unit: Analysis of incidence, risk factors and mortality. Indian J Crit Care Med. 2014;18:200-4

15. Deshmukh B, Kadam S, Thirumugam M, Rajesh K. Clinical study of ventilator-associated pneumonia in tertiary care hospital, Kolhapur, Maharashtra, India. Int J Res Med Sci 2017; 5: 2207-11.

16. Dr. Vinit Garg, Dr. (Col) V.R.R. Chari, Dr. Arnab Paul, Dr. BhoomiRaval, Dr. SoumyanathMaiti, A Study of Ventilator Associated Pneumonia (VAP) in Intensive Care Unit (ICU) setting, Indian Journal of Applied Research, Volume 7(1) JANUARY 2017.

17. SarojGolia, Sangeetha K T, Vasudha C $\mathrm{L}$, Microbial profile of Early and late onset VAP, journal of clinical and diagnostic research ,2013,7(11):24622466.

18. Usman SM, James PM, Rashmi M. Clinical and microbiological facets of ventilator associated pneumonia in the main stream with a practical contact. Int J Res Med Sci 2014; 2: 239-45.

19. Dube M, Goswami S, Singh A, Raju BM, Dube P, Bhatia GC. Pattern and incidence of ventilator associated pneumonia among mechanically ventilated patients. Int $\mathrm{J} \mathrm{Adv}$ Med 2018; 5: 442-5.

20. Maqbool M, Shabir A, Naqash H, Amin A, Koul RK, Shah PA. Ventilator Associated Pneumonia-Incidence and Outcome in Adults in Medical Intensive Care Unit of a Tertiary Care Hospital of North India. Int J Sci Stud 2017; 4(10): 73-76.

21. Mathai AS, Phillips A, Isaac R. 
Ventilator associated pneumonia: A persistent healthcare problem in Indian Intensive Care Units! Lung India 2016; 33: 512-6.

22. Ranjan N, Chaudhary U, Chaudhry D, Ranjan KP. Ventilator-associated pneumonia in a tertiary care Intensive Care Unit: Analysis of incidence, risk factors and mortality. Indian J Crit Care Med. 2014;18:200-4

23. Masih SM, Goel S, Singh A, Tank R, Khichi SK, Singh S. Incidence and risk factors associated with development of ventilator-associated pneumonia from a tertiary care center of northern India. Int J Res Med Sci 2016; 4: 1692-7.

24. Husain Shabbir Ali, Fahmi Yousef Khan, Saibu George, Nissar Shaikh, and Jameela Al-Ajmi, "Epidemiology and Outcome of Ventilator-Associated Pneumonia in a Heterogeneous ICU Population in Qatar," BioMed Research International, vol. 2016, Article ID 8231787, 8 pages,

25. Goel V, Hogade SA, Karadesai SG.
Ventilator associated pneumonia in a medical intensive care unit: Microbial aetiology, susceptibility patterns of isolated microorganisms and outcome. Indian J Anaesth 2012; 56: 558-62.

26. Gupta A, Agrawal A, Mehrotra S, Singh A, Malik S, Khanna A. Incidence, risk stratification, antibiogram of pathogens isolated and clinical outcome of ventilator associated pneumonia. Indian $\mathrm{J}$ Crit Care Med 2011; 15: 96-101.

27. Kant R, Dua R, Beg MA, Chanda R, Gambhir IS, Barnwal S. Incidence, microbiological profile and early outcomes of ventilator associated pneumonia in elderly in a Tertiary Care Hospital in India. Afr J Med Health Sci 2015; 14: 66-9.

28. Patil HV, Patil VC. Incidence, bacteriology, and clinical outcome of ventilator-associated pneumonia at tertiary care hospital. J Nat ScBiol Med 2017; 8: 46-55.

\section{How to cite this article:}

Sadiya Fatima, S. Rajeshwar Rao, V.V. Shailaja and Nagamani, K. 2019. Finding the Incidence of Ventilator Associated Pneumonia by Recent NHSN Guidelines and Its Bacteriological Profile: A Study Conducted in a Tertiary Care Hospital in Southern India. Int.J.Curr.Microbiol.App.Sci. 8(10): 2080-2089. doi: https://doi.org/10.20546/ijcmas.2019.810.242 